\title{
Front Matter: Volume 11314
}

, "Front Matter: Volume 11314," Proc. SPIE 11314, Medical Imaging 2020:

Computer-Aided Diagnosis, 1131401 (21 May 2020); doi: 10.1117/12.2570720

SPIE. Event: SPIE Medical Imaging, 2020, Houston, Texas, United States 


\title{
PROGRESS IN BIOMEDICAL OPTICS AND IMAGING
}

\section{Medical Imaging 2020 \\ Computer-Aided Diagnosis}

\author{
Horst K. Hahn \\ Maciej A. Mazurowski \\ Editors
}

\section{6-19 February 2020}

Houston, Texas, United States

Sponsored by

SPIE

Cooperating Organizations

AAPM-American Association of Physicists in Medicine (United States)

MIPS - Medical Image Perception Society (United States)

SIIM-Society for Imaging Informatics in Medicine (United States)

IFCARS - International Foundation for Computer Assisted Radiology and Surgery (Germany)

WMIS-World Molecular Imaging Society

Published by

SPIE

\section{Volume 11314}

Part One of Two Parts 
The papers in this volume were part of the technical conference cited on the cover and title page. Papers were selected and subject to review by the editors and conference program committee. Some conference presentations may not be available for publication. Additional papers and presentation recordings may be available online in the SPIE Digital Library at SPIEDigitalLibrary.org.

The papers reflect the work and thoughts of the authors and are published herein as submitted. The publisher is not responsible for the validity of the information or for any outcomes resulting from reliance thereon.

Please use the following format to cite material from these proceedings:

Author(s), "Title of Paper," in Medical Imaging 2020: Computer-Aided Diagnosis, edited by Horst K. Hahn, Maciej A. Mazurowski, Proceedings of SPIE Vol. 11314 (SPIE, Bellingham, WA, 2020) Seven-digit Article CID Number.

ISSN: $1605-7422$

ISSN: 2410-9045 (electronic)

ISBN: 9781510633957

ISBN: 9781510633964 (electronic)

Published by

SPIE

P.O. Box 10, Bellingham, Washington 98227-0010 USA

Telephone +13606763290 (Pacific Time) · Fax +1 3606471445

SPIE.org

Copyright @ 2020, Society of Photo-Optical Instrumentation Engineers.

Copying of material in this book for internal or personal use, or for the internal or personal use of specific clients, beyond the fair use provisions granted by the U.S. Copyright Law is authorized by SPIE subject to payment of copying fees. The Transactional Reporting Service base fee for this volume is $\$ 21.00$ per article (or portion thereof), which should be paid directly to the Copyright Clearance Center (CCC), 222 Rosewood Drive, Danvers, MA 01923. Payment may also be made electronically through CCC Online at copyright.com. Other copying for republication, resale, advertising or promotion, or any form of systematic or multiple reproduction of any material in this book is prohibited except with permission in writing from the publisher. The CCC fee code is $1605-$ $7422 / 20 / \$ 21.00$.

Printed in the United States of America by Curran Associates, Inc., under license from SPIE.

Publication of record for individual papers is online in the SPIE Digital Library.

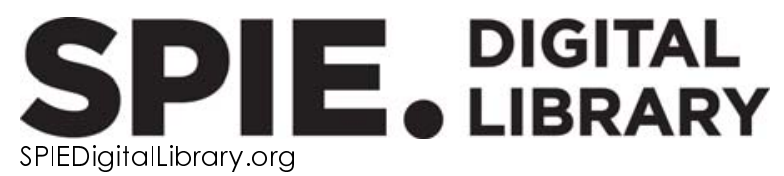

Paper Numbering: Proceedings of SPIE follow an e-First publication model. A unique citation identifier (CID) number is assigned to each article at the time of publication. Utilization of CIDs allows articles to be fully citable as soon as they are published online, and connects the same identifier to all online and print versions of the publication. SPIE uses a seven-digit CID article numbering system structured as follows:

- The first five digits correspond to the SPIE volume number.

- The last two digits indicate publication order within the volume using a Base 36 numbering system employing both numerals and letters. These two-number sets start with 00, 01, 02, 03, 04, 05, 06, 07, 08, 09, OA, OB ... 0Z, followed by 10-1Z, 20-2Z, etc. The CID Number appears on each page of the manuscript. 


\title{
Contents
}

\author{
xiii Authors \\ xix Conference Committee \\ xxiii 2020 Medical Imaging Award Recipients
}

\section{Part One}

\section{MAMMOGRAPHY}

$1131403 \quad$ Microcalcification localization and cluster detection using unsupervised convolutional autoencoders and structural similarity index [11314-2]

1131404 Performance deterioration of deep neural networks for lesion classification in mammography due to distribution shift: an analysis based on artificially created distribution shift [11314-3]

1131405 A multitask deep learning method in simultaneously predicting occult invasive disease in ductal carcinoma in-situ and segmenting microcalcifications in mammography [11314-4]

1131406 Adaptation of a deep learning malignancy model from full-field digital mammography to digital breast tomosynthesis [11314-5]

\section{CHEST I}

$1131407 \quad$ Fast few-shot transfer learning for disease identification from chest $x$-ray images using autoencoder ensemble [11314-6]

1131408 Weakly supervised 3D classification of chest CT using aggregated multi-resolution deep segmentation features [11314-7]

1131409 Bone suppression on chest radiographs with adversarial learning [11314-8]

$113140 \mathrm{~A}$ Cascade of U-Nets in the detection and classification of coronary artery calcium in thoracic low-dose CT [11314-9]

$11314 \mathrm{OB}$ Comparison of CNN architectures and training strategies for quantitative analysis of idiopathic interstitial pneumonia [11314-10]

$113140 C$ Deep learning for pneumothorax detection and localization using networks fine-tuned with multiple institutional datasets [11314-11] 
11314 OD Combining symmetric and standard deep convolutional representations for detecting brain hemorrhage [11314-12]

$11314 \mathrm{OE} \quad$ Generative synthetic adversarial network for internal bias correction and handling class imbalance problem in medical image diagnosis [11314-13]

11314 OF Automatic detection of contrast enhancement in T1-weighted brain MRI of human adults [11314-14]

$113140 G \quad$ A hyperacute stroke segmentation method using 3D U-Net integrated with physicians' knowledge for NCCT [11314-15]

$11314 \mathrm{OH} \quad$ Deep learning with context encoding for semantic brain tumor segmentation and patient survival prediction [11314-16]

\section{ABDOMEN}

$11314 \mathrm{Ol} \quad$ Organ segmentation from full-size CT images using memory-efficient FCN [11314-17]

11314 OK Multilevel UNet for pancreas segmentation from non-contrast CT scans through domain adaptation [11314-19]

11314 OL Robust hepatic vessels segmentation model based on noisy dataset [11314-20]

$113140 \mathrm{M}$ A combination of intra- and peri-tumoral deep features from prostate bi-parametric MRI can distinguish clinically significant and insignificant prostate cancer [11314-21]

$113140 \mathrm{~N}$ Integration of optical and virtual colonoscopy images for enhanced classification of colorectal polyps [11314-22]

\section{MUSCULOSKELETAL}

$1131400 \quad$ Accurately identifying vertebral levels in large datasets [11314-23]

11314 OP Semi-supervised learning for predicting total knee replacement with unsupervised data augmentation [1 1314-24]

$113140 Q \quad$ Deciphering tissue relaxation parameters from a single MR image using deep learning [1 1314-25]

11314 OR Automatic Kellgren-Lawrence grade estimation driven deep learning algorithms [11314-26]

11314 OS Computer-aided detection of focal bone metastases from whole-body multi-modal MRI [1 1314-27] 
Proc. of SPIE Vol. 11314 1131401-5 Downloaded From: https://www.spiedigitallibrary.org/conference-proceedings-of-spie on 25 Apr 2023
Terms of Use: https://www.spiedigitallibrary.org/terms-of-use 


\section{RADIOMICS}

11314 OT U-radiomics for predicting survival of patients with idiopathic pulmonary fibrosis [11314-28]

$113140 \mathrm{OU}$ Dependence of radiomics features on CT image acquisition and reconstruction parameters using a cadaveric liver [11314-29]

$113140 \mathrm{~V}$ Multi-site evaluation of stable radiomic features for more accurate evaluation of pathologic downstaging on MRI after chemoradiation for rectal cancers [11314-30]

11314 OW Robust radiomic feature selection in digital mammography: understanding the effect of imaging acquisition physics using phantom and clinical data analysis [1 1314-31]

$113140 X \quad$ Improvement of classification performance using harmonization across field strength of radiomic features extracted from DCE-MR images of the breast [11314-33]

11314 OY Machine learning-powered prediction of recurrence in patients with non-small cell lung cancer using quantitative clinical and radiomic biomarkers [11314-32]

BREAST MRI, SKIN

$113140 Z$ Explainable Al for medical imaging: deep-learning CNN ensemble for classification of estrogen receptor status from breast MRI [11314-52]

1131410 Deep learning predicts breast cancer recurrence in analysis of consecutive MRIs acquired during the course of neoadjuvant chemotherapy [11314-34]

$1131411 \quad$ Using ResNet feature extraction in computer-aided diagnosis of breast cancer on 927 lesions imaged with multiparametric MRI [1 1314-35]

1131412 Interpretable deep learning regression for breast density estimation on MRI [1 1314-69]

$1131413 \quad$ MRI image harmonization using cycle-consistent generative adversarial network [11314-36]

1131414 Melanoma detection with electrical impedance spectroscopy and dermoscopy using joint deep learning models [11314-37]

1131415 A multidimensional scaling and sample clustering to obtain a representative subset of training data for transfer learning-based rosacea lesion identification [1 1314-38]

\section{BREAST}

$1131416 \quad$ Hazards of data leakage in machine learning: a study on classification of breast cancer using deep neural networks [11314-39] 
1131417 Architectural distortion detection approach guided by mammary gland spatial pattern in digital breast tomosynthesis [11314-40]

1131418 Simulating breast mammogram using conditional generative adversarial network: application towards finding mammographically-occult cancer [11314-41]

1131419 Weakly-supervised US breast tumor characterization and localization with a box convolution network [11314-42]

$113141 \mathrm{~A}$ Performance comparison of different loss functions for digital breast tomosynthesis classification using 3D deep learning model [11314-43]

\section{CHEST II, LYMPH NODES}

$113141 \mathrm{~B} \quad$ Automated detection and segmentation of mediastinal and axillary lymph nodes from CT using foveal fully convolutional networks [1 1314-44]

11314 1C Feasibility of end-to-end trainable two-stage U-Net for detection of axillary lymph nodes in contrast-enhanced CT based on sparse annotations [11314-45]

$11314 \mathrm{lE} \quad$ Lung vessel suppression and its effect on nodule detection in chest CT scans [11314-46]

$11314 \mathrm{lF} \quad$ Hybrid deep-learning model for volume segmentation of lung nodules in CT images [11314-47]

$113141 \mathrm{H} \quad$ EDICNet: An end-to-end detection and interpretable malignancy classification network for pulmonary nodules in computed tomography [11314-49]

\section{KEYNOTE AND METHODOLOGY}

$113141 \mathrm{~J}$ Weakly-supervised lesion segmentation on CT scans using co-segmentation [11314-51]

$113141 \mathrm{~K} \quad$ Quality controlled segmentation to aid disease detection [11314-138]

HEAD AND NECK, EYE

$113141 \mathrm{~L} \quad$ Spatio-spectral deep learning methods for in-vivo hyperspectral laryngeal cancer detection [11314-53]

$113141 \mathrm{M}$ Decision fusion on image analysis and tympanometry to detect eardrum abnormalities [1 1314-54]

$113141 \mathrm{~N}$ Direct classification of type 2 diabetes from retinal fundus images in a population-based sample from the Maastricht study [11314-55] 
1131410 Segmentation of retinal low-cost optical coherence tomography images using deep learning [11314-56]

NOVEL APPLICATIONS

$113141 \mathrm{P} \quad$ Attention-guided classification of abnormalities in semi-structured computed tomography reports [11314-57]

$113141 Q \quad$ Cascading YOLO: automated malaria parasite detection for Plasmodium vivax in thin blood smears [1 1314-58]

$113141 R \quad$ Segmentation of uterus and placenta in MR images using a fully convolutional neural network [11314-59]

1131415 A multi-stage fusion strategy for multi-scale GLCM-CNN model in differentiating malignant from benign polyps [11314-60]

$113141 \mathrm{~T} \quad$ Convolutional neural network-based decision support system for bladder cancer staging in CT urography: decision threshold estimation and validation [11314-61]

NEURO II

$113141 \mathrm{~A} \quad$ Attention-deficit/hyperactivity disorder prediction using graph convolutional networks [1 1314-62]

$113141 \mathrm{~V} \quad$ An extended-2D CNN for multiclass Alzheimer's Disease diagnosis through structural MRI [11314-63]

$11314 \mathrm{lW} \quad$ Combining deep and hand-crafted MRI features for identifying sex-specific differences in autism spectrum disorder versus controls [11314-65]

$113141 \mathrm{X} \quad$ Multi-modal deep learning for predicting progression of Alzheimer's disease using bi-linear shake fusion [11314-66]

$113141 Y \quad$ Large-scale Extended Granger Causality (IsXGC) for classification of Autism Spectrum Disorder from resting-state functional MRI [11314-64]

$1131412 \quad$ Prognostic power of the human psoas muscles FDG metabolism in amyotrophic lateral sclerosis [11314-67]

\section{POSTER SESSION}

1131420 DCGANs for realistic breast mass augmentation in x-ray mammography [11314-68] 
$1131421 \quad$ Case-based repeatability of machine learning classification performance on breast MRI [11314-70]

1131422 Genetic algorithm for machine learning architecture selection for breast MRI classification [11314-71]

1131423 Automated breast cancer risk estimation on routine CT thorax scans by deep learning segmentation [11314-72]

1131424 Automatic detection and classification of abnormal tissues on digital mammograms based on a bag-of-visual-words approach [11314-73]

1131426 Generating high resolution digital mammogram from digitized film mammogram with conditional generative adversarial network [11314-75]

\section{Part Two}

1131428 Generative adversarial network-based image completion to identify abnormal locations in digital breast tomosynthesis images [11314-77]

1131429 3D U-Net for segmentation of pulmonary nodules in volumetric CT scans from multi-annotator truth estimation [1 $1314-78]$

$113142 \mathrm{~A} \quad$ Weak supervision in convolutional neural network for semantic segmentation of diffuse lung diseases using partially annotated dataset [1 1314-79]

$113142 \mathrm{~B} \quad$ False positive reduction of vasculature for pulmonary nodule detection [11314-80]

$113142 \mathrm{C} \quad$ Multi-task learning for mortality prediction in LDCT images [11314-81]

$113142 \mathrm{D} \quad$ Association analysis of SNPs with CT image-based phenotype of emphysema progression in heavy smokers [1 1314-82]

$113142 \mathrm{E} \quad$ Active semi-supervised expectation maximization learning for lung cancer detection from Computerized Tomography (CT) images with minimally label training data [11314-83]

$113142 \mathrm{~F} \quad$ Artificially augmenting data or adding more samples? A study on a 3D CNN for lung nodule classification [1 1314-84]

$113142 \mathrm{G}$ Assessment of CT image reconstruction parameters on radiomic features in a lung cancer screening cohort: the PROSPR study [11314-85]

$1131421 \quad$ Deep learning methods for segmentation of lines in pediatric chest radiographs [11314-87]

$113142 \mathrm{~J} \quad$ Differential diagnosis of pulmonary nodules using 3D CT images [1 1314-88] 
$113142 \mathrm{~K} \quad$ Radiomics-based texture analysis of idiopathic pulmonary fibrosis for genetic and survival predictions [11314-89]

$113142 \mathrm{~L} \quad$ Lung tumor segmentation using coupling-net with shape-focused prior on chest CT images of non-small cell lung cancer patients (Cum Laude Poster Award) [11314-90]

$113142 \mathrm{M} \quad$ Deep convolutional neural networks for molecular subtyping of gliomas using magnetic resonance imaging [11314-91]

1131420 Predicting treatment outcome of intracranial aneurysms using angiographic parametric imaging and recurrent neural networks [11314-93]

$113142 \mathrm{R} \quad$ Prediction of $\mathrm{MCl}$ to $\mathrm{AD}$ risk of conversion survival models: $q M \mathrm{MI}$ vs CSF measures and cognitive assessments [11314-96]

$1131425 \quad$ Diagnosis of Parkinson's Disease with a hybrid feature selection algorithm based on a discrete artificial bee colony [11314-97]

$113142 \mathrm{~T} \quad$ Advanced magnetic resonance imaging based algorithm for local grading of glioma [1 1314-98]

$113142 \mathrm{U} \quad$ Neural networks for in situ detection of glioma infiltration using optical coherence tomography [1 1314-99]

$113142 \mathrm{~V} \quad$ A data-driven approach for stratifying psychotic and mood disorders subjects using structural magnitude resonance imaging data [11314-100]

$113142 \mathrm{~W} \quad$ Multi-resolution CNN for brain vessel segmentation from cerebrovascular images of intracranial aneurysm: a comparison of U-Net and DeepMedic [11314-101]

$113142 X \quad$ Automatic detection of brain metastases using 3D mask R-CNN for stereotactic radiosurgery [11314-102]

$113142 Y \quad$ Automatic brain arteriovenous malformations segmentation on contrast CT images using combined region proposal network and V-Net [11314-103]

$113142 Z$ Computer-assisted quantification of surgical outcome in infants with sagittal craniosynostosis in 3D head CT images using mean normal skull model [11314-104]

1131430 "Lesion-habitat" radiomics to distinguish radiation necrosis from fumor recurrence on posttreatment MRI in metastatic brain tumors [11314-105]

$1131431 \quad$ First steps into endoscopic video analysis for Barrett's cancer detection: challenges and opportunities [11314-106]

1131433 Decision fusion of 3D convolutional neural networks to triage patients with suspected prostate cancer using volumetric biparametric MRI [11314-108]

1131434 Visualizing intestines for diagnostic assistance of ileus based on intestinal region segmentation from 3D CT images [11314-109] 
1131435 Comparative performance of 3D-DenseNet, 3D-ResNet, and 3D-VGG models in polyp detection for CT colonography [11314-110]

1131436 Machine learning methods to predict presence of intestine damage in patients with Crohn's disease [1 1314-1 11 ]

1131437 The field effect in Barrett's Esophagus: a macroscopic view using white light endoscopy and deep learning [1 1314-112]

1131438 Visualising decision-reasoning regions in computer-aided pathological pattern diagnosis of endoscytoscopic images based on CNN weights analysis [11314-113]

1131439 Computer-aided staging of gastric cancer using radiomics signature on computed tomography imaging [11314-114]

$113143 \mathrm{~A} \quad$ Automatic polyp detection and localization during colonoscopy using convolutional neural networks [11314-115]

$113143 \mathrm{~B} \quad$ Performance investigation of deep learning vs. classifier for polyp differentiation via texture features [11314-116]

$113143 \mathrm{C}$ Comparative performance of 3D machine-learning and deep-learning models in the detection of small polyps in dual-energy CT colonography [11314-117]

$113143 \mathrm{D} \quad$ A deep learning based integration of multiple texture patterns from intensity, gradient and curvature GLCMs in differentiating the malignant from benign polyps [11314-118]

11314 3E Deformation robust texture features for polyp classification via CT colonography [11314-119]

$113143 \mathrm{~F} \quad$ Evaluating texture-based prostate cancer classification on multi-parametric magnetic resonance imaging and prostate specific membrane antigen positron emission tomography [11314-120]

$113143 G \quad$ Radiomic features derived from periprostatic fat on pre-surgical T2w MRI predict extraprostatic extension of prostate cancer identified on post-surgical pathology: preliminary results [11314-121]

$113143 \mathrm{H} \quad$ Automatic liver segmentation in abdominal CT images using combined 2.5D and 3D segmentation networks with high-score shape prior for radiotherapy treatment planning [11314-122]

$1131431 \quad$ Prediction of prostate cancer aggressiveness using quantitative radiomic features using multiparametric MRI [11314-123]

$113143 \mathrm{~J} \quad$ Renal parenchyma segmentation in abdominal CT images based on deep convolutional neural networks with similar atlas selection and transformation [11314-124]

$113143 \mathrm{~K} \quad$ Bladder wall segmentation using U-net based deep learning [11314-125]

$113143 \mathrm{~L} \quad$ Survival prediction of liver cancer patients from CT images using deep learning and radiomic feature-based regression [11314-126] 
$113143 \mathrm{M} \quad \mathrm{CNN}$-based detection of distal tibial fractures in radiographic images in the setting of open growth plates [11314-127]

$113143 \mathrm{~N} \quad$ Automatic estimation of knee joint space narrowing by deep learning segmentation algorithms [11314-128]

$1131430 \quad$ Knee orientation detection in MR scout scans using 3D U-net [11314-129]

$113143 \mathrm{P} \quad$ Classification of lesion specific myocardial ischemia using cardiac computed tomography radiomics [11314-130]

$113143 Q \quad$ Siamese neural networks for the classification of high-dimensional radiomic features [11314-131]

$113143 R \quad$ Survey of image denoising methods for medical image classification [11314-132]

11314 3Т Evaluating several ways to combine handcrafted features-based system with a deep learning system using the LUNA16 Challenge framework [11314-134]

$113143 \mathrm{U} \quad$ A post-acquisition standardization method for positron emission tomography images [1 1314-135]

$113143 \mathrm{~V} \quad$ Usefulness of fine-tuning for deep learning based multi-organ regions segmentation method from non-contrast CT volumes using small training dataset [11314-136]

11314 3W Synthesize CT from paired MRI of the same patient with patch-based generative adversarial network [1 1314-137]

$113143 \mathrm{X} \quad$ Multi-modal component subspace-similarity-based multi-kernel SVM for schizophrenia classification [11314-139]

$113143 Y \quad$ The efficacy of microaneurysms detection with and without vessel segmentation in color retinal images [11314-140]

$1131432 \quad$ Cup-disc and retinal nerve fiber layer features fusion for diagnosis glaucoma [11314-141]

$1131440 \quad$ Benign and malignant thyroid classification using computed tomography radiomics [11314-142]

$1131441 \quad$ Automatic classification of carotid ultrasound images based on convolutional neural network [11314-143]

$1131443 \quad$ Verification of accuracy of an algorithmic image-based dental pulp vitality test [11314-145]

1131444 Organ-at-Risk (OAR) segmentation in head and neck CT using U-RCNN [11314-146]

1131445 3D thyroid segmentation in CT using self-attention convolutional neural network [11314-147]

1131446 Automated eye disease classification method from anterior eye image using anatomical structure focused image classification technique [11314-148] 
$1131447 \quad$ Hyperparameter selection for ResNet classification of malignancy from thyroid ultrasound images [11314-149]

1131448 Detecting age-related macular degeneration (AMD) biomarker images using MFCC and texture features [1 1314-150]

$113144 \mathrm{~A} \quad$ Radiomics and artificial intelligence analysis of CT data for the identification of prognostic features in multiple myeloma [11314-152]

11314 4B Automated discomfort detection for premature infants in NICU using time-frequency featureimages and CNNs [11314-153]

11314 4C Standardization of blood flow measurements by automated vascular analysis from power Doppler ultrasound scan [11314-154]

$113144 \mathrm{D} \quad$ Investigation of the accuracy of classifying coronary artery disease severity using machine learning with subdomain analysis of fractional flow reserve diagnosis in patients [11314-156]

$113144 \mathrm{E} \quad$ Investigation of sex hormones on the early diagnosis of schizophrenia [11314-157]

11314 4F Mask R-CNN based coronary artery segmentation in coronary computed tomography angiography [11314-158]

$113144 \mathrm{G} \quad$ Fully automated spectral envelope and peak velocity detection from Doppler echocardiography images [11314-159] 


\section{Authors}

Numbers in the index correspond to the last two digits of the seven-digit citation identifier (CID) article numbering system used in Proceedings of SPIE. The first five digits reflect the volume number. Base 36 numbering is employed for the last two digits and indicates the order of articles within the volume. Numbers start with 00, 01, 02, 03, 04, 05, 06, 07, 08, 09, 0A, 0B...0Z, followed by 10-1Z, 20-2Z, etc.

Aarts, Ronald M., 4B

Abbasi, Almas, ON

Abe, Hiroyuki, 21

Aberle, Denise R., $1 \mathrm{H}$

Acciavatti, Raymond J., OW

Adams, Joe, 47

Agarwal, Vatsal, $1 \mathrm{~J}$

Ahmed, Hashim, 33

Akahori, Sadato, $0 G$

Alessio, Adam M., 22, 21, 47

Alfano, R., 3F

Al-Hallaq, Hania, OU

Al-Hawary, Mahmoud M., 36

Almohsen, Ranya, 06

Altun, Hidir Cem, 1C

Alva, Ajjai, 1T, 3K

Alyafi, Basel, 20

Amano, Hizuru, 34

Amini, Amir, 29

Andriessen, Peter, 4B

Angel, Erin, 4D

Annapragada, Ananth, 3M

Antani, Sameer, 1Q, 4G

Antonelli, Michela, 33

Antunes, Jacob, 0V, IW

Aokage, Yoshiki, 2J

Aoki, Shigeki, 3V

Armato, Samuel G. III, OU, 2K

Baladandayuthapani, Veera, $2 \mathrm{~T}$

Banerjee, Anushka, oN

Barish, Matthew, ON

Barman, Arko, OD

Bauckneht, Matteo, 12

Bauman, G.S., 3F

Beitler, Jonathan J., 44

Bengs, Marcel, 14, $1 \mathrm{~L}$

Bequette, Justin, 43

Bera, Kaustav, OV

Berendschot, Tos T. J. M., IN

Berg, Wendie, 1 A

Bergman, Jacques, 31

Bertels, Jeroen, 23

Betz, Christian, $1 \mathrm{~L}$

Bhatia, Parmeet, 30

Bi, Sarah, 43

Bignotti, Bianca, 4A

Bingmer, Katherine, OV

Binol, Hamidullah, 15, $1 \mathrm{M}$

Biswas, Debosmita, 22
Bogoni, Luca, 2B

Bosmans, Hilde, 23

Brady, Justin T., OV

Braman, Nathaniel, OM

Bravo, Diego, 3A

Brillet, Pierre-Yves, OB

Brosch, Tom, 1B

Bucobo, Juan Carlos, ON

Buda, Mateusz, 13, 28, 3N

Budzikowski, Jorie D., 2K

Burkow, Jonathan, 21

Butler, J., 3F

Byun, Sohyun, $2 \mathrm{~L}$

Calhoun, Vince D., 2V, 3X

Campi, Cristina, 1Z, 4A

Cao, Weiguo, 1S, 3D, 3E

Caoili, Elaine M., 1T, 3K

Caponnetto, Claudia, $1 \mathrm{Z}$

Carolus, Heike, 1B

Carras, Peter S., 22

Cea, Michele, 4A

Ceranka, Jakub, OS

Cha, Kenny H., 04

Chaichana, Kaisorn L., $2 \mathrm{U}$

Chan, Heang-ping, 0Z, 16, 1F, 1T, 26, 3K

Chang, Gregory, OP

Chapman, David, 2E

Chapman-Sung, Daniel Hoklai, $1 T$

Chen, Deji, 3Q

Chen, Jonathan, 30

Chen, Weiguo, 17

Cheng, Xinyao, 41

Chin, J. L., 3F

Chiò, Adriano, 12

Chlebus, Grzegorz, 1C

Cho, Kyunghyun, OP

Choi, Dahim, OY

Choi, Jang-Hwan, OY

Choi, Yeseul, 3B

Chughtai, Aamer, IF

Chung, Jonathan $\mathrm{H}$., $2 \mathrm{~K}$

Cistaro, Angelina, $1 \mathrm{Z}$

Cohan, Richard H., 1T, 3K

Cohen, Eric A., OW, 2G

Collins, Sally L., 4C

Conant, Emily F., OW

Conte, Alessio, 4A

Correa, Ramon, 30

Cossu, Vanessa, 1 Z 
Cox, Joseph, 47

Crosby, Jennie, OC

Curran, Walter J., 2X, 2Y, 40, 44, 45, 4F

Curvers, Wouter, 31

Dai, Qionghai, 25

D'Angelo, William, 43

D'Anniballe, Vincent M., 08, 1P

Davies, Jason M., 20

De Buck, Stijn, 23

de Groof, Jeroen, 31, 37

de Mey, Johan, OS

de With, Peter H. N., 31, 37, 4B

Deniz, Cem M., OP

Dewaele, Tanguy, 23

Diaz, Oliver, 20

Dighe, Manjiri, 47

Ding, Mingyue, 41

Do, Quyen N., 1R

Doganay, Emine, $1 \mathrm{~A}$

Dominietto, Alida, 4A

Dong, Xue, 44

Dormer, James D., 1R, 3Q

Drukker, Karen, 10, 21

Du, Jiang, $0 Q$

Edwards, Alexandra, 10

Eggert, Dennis, $1 \mathrm{~L}$

Eguchi, Kenji, 2J

Elmaraghy, Charles, IM

Elton, Daniel C., 00

Emberton, Mark, 33

Enchakalody, Binu E., 36

Essig, Garth, 1M

Falakshahi, Haleh, 2V

Fan, James Z., OD

Fang, Qiming, 1E

Fantini, Irene, 1V

Faryna, Khrystyna, 08, 1P

Fawzi, Amani A., 48

Fei, Baowei, 1R, 3Q

Fenster, Aaron, 41

Fetita, Catalin, $\mathrm{OB}$

Foxe, John J., 1U, 1Y

Foy, Joseph J., OU, 2K

Frassoni, Francesco, 4A

Friedman, Kenneth, OV

Frigui, Hichem, 29

Fu, Yabo, 2Y, 4F

Fuchigami, Takuya, OG

Fuentes, David T., 2T

Fuhrman, Jordan D., OA

Fukuoka, Hideki, 46

Fuller, Gregory N., 2T

Funke, William, 29

Furst, Jacob, 48

Gaed, M., 3F

Gandikota, Dhanuj, IT

Gao, Hao, $2 S$

Gao, Long, 1A

Gao, Shuang, 3X
Gao, Yongfeng, 1S, 3D

Garcia-Naranjo, Juan, 3T

Gastounioti, Aimilia, OW

Gates, Evan D. H., 2T

Geha, Paul, $1 Y$

Geng, Xiujuan, 4E

Gertsenshteyn, Inna H., OU

Gessert, Nils, 14, $1 \mathrm{~L}$

Ghate, Sujata V., 28

Giancardo, Luca, OD

Giger, Maryellen L., OA, OC, 0X, 10, 11, 21

Gilhuijs, Kenneth G. A., 12

Gomez, J. A., 3F

Gómez, Martín, 3A

Gonidakis, Panagiotis, 2F, 3T

Gossmann, Alexej, 04

Goto, Tsubasa, 1X

Grimm, Lars J., 03, 05

Gu, Xiaomeng, 1E

Gualco, Stefano, 4A

Guo, Bang Jun, 3P, 40, 45, 4F

Guo, Hengtao, 2C

Guo, Yanhui, 17

Gurcan, Metin N., 15, $1 \mathrm{M}$

Hadjiiski, Lubomir M., 0Z, 16, 1F, 1T, 26, 3K

Haghighi, Babak, 2G

Hahn, Horst K., 1C

Hamilton, Jackson, 2T

Han, Simon X., $1 \mathrm{H}$

Handels, Heinz, 10

Hansen, Colin B., 2B

Harms, Joseph, 44

Hashimoto, Masahiro, 3V

Hayashi, Yuichiro, 3V

Hazle, John D., 2T

$\mathrm{He}$, Xiuxiu, 3P, 40, 45

$\mathrm{He}$, Zilong, 17

Heinrich, Mattias P., 10

Helvie, Mark A., 0Z, 26

Henderson, Brianna, 36

Henry, Ronald M. A., $1 \mathrm{~N}$

Henschke, Claudia I., OA

Heslinga, Friso G., $1 \mathrm{~N}$

Hiremath, Amogh, OM

Hiremath, Yashas, $1 \mathrm{~W}$

Hironaka, Toru, OT, 35

Höink, Anna, 1B

Holste, Greg, 21

Hong, Helen, OY, 2L, 2Z, 3H, 3I, 3J, 3L

Hou, Rui, 03, 05, 08, IP

Houben, A. J. H. M., IN

Hsieh, Meng-Kang, OW

Hsu, Li-Yueh, 4G

Hsu, William, $1 \mathrm{H}$

Hu, Qiyuan, 11

Huo, Yumei, $1 \mathrm{~S}$

Hüttmann, Gereon, 10

Hwang, E. Shelley, 03, 05

Hwang, Sung II, 3I 
Iftekharuddin, Khan M., $\mathrm{OH}$

Imoto, Issei, 2D

Ionita, Ciprian N., 2O, 4D

Ishii, Genichirou, 2J

Ismail, Marwa, IW

Itoh, Hayato, 34, 38

Iuga, Andra-Iza, 1B

Ivanitskiy, Michael, 3K

lyer, Vijay, 4D

Jacobs, Colin, 1C

Jaeger, Stefan, $1 Q$

Jaiswal, Prakhar, 2W

Janse, Markus H. A., 12

Jansen, Bart, 2F, 3T

Jeong, Taesik, $3 \mathrm{H}$

Jerebko, Anna, 2B

Jiang, Xiaojun, $2 Y$

Jinzaki, Masahiro, 3V

Jiranek, William A., OR, 3N

Jirapatnakul, Artit C., OA

Jo, Javier, $2 \mathrm{U}$

Juarez-Chambi, Ronald M., $2 U$

Jung, Julip, 2L, 3H, 3l

Kaffenberger, Benjamin H., 15

Kageyama, Takeru, 2J

Kahn, Shannon, 2X

Kalady, Matthew, oV

Kalra, Mannudeep K., 2C

Kan, J. Herman, 3M

Kaneko, Masahiro, 2D, 2J

Kang, Hong, $3 Z$

Karargyris, Alexandros, $1 \mathrm{~K}$

Kawata, Yoshiki, 2D, 2J

Kazerooni, Ella A., $1 \mathrm{~F}$

Kepp, Timo, 10

Kerlikowske, Karla, ow

Kido, Shoji, 2A

Kim, Bong-Seog, OY, 2L

Kim, Chanho, 19

Kim, Hye Jung, 19

Kim, Hyeonjin, 3J

Kim, Hyoungseop, 0T, 35, 3C

Kim, Jaeil, 19

Kim, Jin Sung, 3H, 3L

Kim, Junmo, 3L

Kim, Myoung Hee, OY

Kim, Se Hyung, 3C

Kim, Won Hwa, 19

Kim, Young-Gi, 3l

King, Lorraine M., 05

Kitasaka, Takayuki, 34

Klinder, Tobias, 1B

Koch, Peter, 10

Kommers, Deedee, 4B

Koneru, Sathvik, 16

Kontos, Despina, 0W, 2G

Kruger, Melanie, 2C

Kudo, Shin-ei, 38

Kumamaru, Kanako K., 3V, 4D
Kusumoto, Masahiko, 2D, 2J

Kut, Carmen, $2 \mathrm{U}$

Laffers, Wiebke, $1 \mathrm{~L}$

Lai, Rita, 1Z, 4A

LaMontagne, Pamela, OF

Lecouvet, Frédéric, OS

Lee, Christoph, 22

Lee, Hak Jong, 31

Lee, Hansang, $3 \mathrm{~L}$

Lee, Ji-Yeon, OY

Lee, Juhun, 18

Lee, Min Jin, $2 Z$

Lee, Songmi, OD

Lei, Qiu, 30

Lei, Yang, 2X, 2Y, 3P, 40, 44, 45, 4F

Levy, Elad I., 20

Lewis, Matthew A., IR

$\mathrm{Li}$, Chen, $3 \mathrm{O}$

Li, Feng, OC

Li, Haolun, $2 S$

Li, Lihong, $1 \mathrm{~S}$

Li, Nianyi, OR, 28, 3N

Li, Puchen, 1A

Li, Qiang, 1E

Li, Qinmei, 3Q

Li, Wen, 4G

$\mathrm{Li}$, Xiaoxing, $3 \mathrm{Z}$

$\mathrm{Li}$, Xingde, $2 \mathrm{U}$

Li, Yan, 3W

Li, Yiming, $2 \mathrm{M}$

Li, Yuanzhong, 0G, $1 \mathrm{X}$

Li, Yue, 17

Liang, David, 3B

Liang, Jia, 09

Liang, Zhengrong, 0N, 1S, 3D, 3E

Lin, Jonathan S., 2T

Lin, Yannan, $1 \mathrm{H}$

Lippert, Christoph, OE

Liu, Li, OL

Liu, Tian, 2X, 2Y, 3P, 40, 44, 45, 4F

Liu, Yingzi, 45

Liu, Ziteng, $2 M$

Lo, Joseph Y., 03, 05, 08, 1P

Long, Aaron, 06

Long, $\mathrm{Xi}, 4 \mathrm{~B}$

Loo, Claudette E., 12

Looney, Pádraig, 4C

Lopez-Rivera, Victor, OD

Lotufo, Roberto, $1 \mathrm{~V}$

LU, Hongbing, 3E

Lu, Yao, 17, 26, 3W

Lu, Zhongyang, 38

Luo, Yahong, $1 \mathrm{~A}$

Ma, Xiangyuan, 17

Ma, Xufan, 48

Ma, Yajun, $0 Q$

MacMahon, Heber, OC

Madabhushi, Anant, OM, OV, $3 G$

Madhuranthakam, Ananth J., IR 
Maghsoudi, Omid Haji, OW

Mahajan, Abhishaike, 3Q

Mahran, Amr, 3G

Maidment, Andrew D. A., OW

Maintz, David, 1B

Maley, Carlo C., 05

Marcus, Daniel, OF

Marini, Cecilia, 12

Marks, Jeffrey R., 03, 05

Marti, Robert, 20

Martinez, Laura, 43

Massone, Anna Maria, 1Z, 4A

Matsuhiro, Mikio, 2D

Matsumoto, Yuji, 2J

Matthews, Thomas Paul, 06

Maude, Richard J., 1Q

Mazurowski, Maciej A., 05, 08, 0R, 13, 28, 3N

Mehdizadeh, Alireza, 3 Y

Mehta, Pritesh, 33

Meine, Hans, $1 \mathrm{C}$

Meinel, Christoph, OE

Meng, Hui, 2W

Menon, Sumeet, 2E

Michael, Peter F., 3R

Milchenko, Mikhail, OF

Misawa, Kazunari, 3V

Misawa, Masashi, 38

Mitsouras, Dimitrios, 4D

Moberly, Aaron C., 1M

Modanwal, Gourav, 13

Mokin, Maxim, 2W

Mombourquette, Brent, 06

Moon, Sehwa, OY

Moradi, Mehdi, $1 \mathrm{~K}$

Morbelli, Silvia, 12

Mori, Kensaku, 0l, 34, 38, 3V, 46

Mori, Yuichi, 38

Morris, Michael, 2E

Mortani Barbosa, Eduardo Jr., 2G

Mortazi, Aliasghar, 3U

Moussa, M., 3F

Mueller, Nina A., $1 \mathrm{~L}$

Nakano, Yasutaka, 2D

Näppi, Janne J., OE, OT, 35, 3C

Nazar, Mahdieh, $3 Y$

Neal, Colleen $\mathrm{H} ., \mathrm{OZ}$

Nguyen, Phuong, 2E

Niazi, M. Khalid Khan, 15, $1 \mathrm{M}$

Niki, Nobory, 2D, 2J

Nishikawa, Robert M., 18

Nishio, Kohei, 34

Noël, Peter B., 2G

Noth, Imre, 2K

O. H. Gerstner, Andreas, $1 \mathrm{~L}$

Oda, Hirohisa, 34

Oda, Masahiro, 0l, 34, 38, 3V, 46

O'Donnell, Jonathan, OR, 3N

Ofshteyn, Asya, OV

Oh, Hoonil, 2L
Ohmatsu, Hironobu, 2J

Okatani, Takayuki, OG

Okemgbo, Abum, 48

Oliva, Diego, 24

Orozco-Sanchez, Jorge, 2R

Ourselin, Sebastien, 33

Paliwal, Nikhil, 2W

Pan, Long Sheng, 2S

Pantalone, Lauren, 0W, $2 \mathrm{G}$

Papaioannou, John, 10

Papanastasopoulos, Zachary, $\mathrm{OZ}$

Paramagul, Chintana, $0 \mathrm{Z}$

Partridge, Savannah, 22

Paspulati, Rajmohan, OV

Patel, Tatsat R., 2W

Paul, Angshuman, 07, OK

Pautler, S., 3F

Pavlosky, W., 3F

Pedemonte, Stefano, 06

Pei, Linmin, $\mathrm{OH}$

Peitzsch, Andrew, 43

Peng, Yifan, 03

Pereira, Carina, 22, 47

Pereira, Mariana, $1 \mathrm{~V}$

Pérez-Cisneros, Marco, 24

Piana, Michele, 1Z, 4A

Pickhardt, Perry J., OK, ON, 0O, 3B, 3E

Plotner, Alisha, 15

Pluim, Josien P. W., $1 \mathrm{~N}$

Podgorsak, Alexander R., 2O, 4D

Pomeroy, Marc J., ON, 3B, 3D, 3E

Ponsky, Lee, 3G

Prabhu, Sujit S., 2T

Prasanna, Prateek, OM

Punwani, Shonit, 33

Purysko, Andrei, OM, OV

Püsken, Michael, 1B

Qi, Zhenyu, 3W

Qian, Tianyi, 2M

Quinones-Hinojosa, Alfredo, 2U

Quizon, Nicolas, $1 Q$

Rachinsky, I., 3F

Ragusi, Max A. A., 12

Rahman, Md Monibor, $\mathrm{OH}$

Rai, Rahul, 2W

Raicu, Daniela, 48

Rashid, Ahmed A., 2K

Rastinehad, Art, OM

Rava, Ryan A., 20

Rein, David, 48

Ren, Yinhao, 03

Rendle, Katharine A., $2 G$

Rennotte, Simon, OB

Rezaei, Mina, OE

Rha, Koon Ho, 3J

Rhines, Thomas, OC

Rittner, Leticia, IV

Rodríguez-Esparza, Erick, 24

Roider, Johann, 10 
Rokham, Hooman, 2V

Romero, Eduardo, 3A

Rossi, Federica, 4A

Roth, Holger R., 3V

Ruano, Josue, 3A

Rubin, Geoffrey D., 08, 1 P

Rubin, Sydney, 47

Rybicki, Frank J., 4D

Saboksayr, Seyed Saman, 1U, 1 Y

Sachdev, Vandana, 4G

Saha, Anindo, 08

Saha, Ashirbani, 28

Saia, Chelsea, 2G

Said, Nicholas, OR, 3N

Samala, Ravi K., 0Z, 16, 1T, 3K

Sambuceti, Gianmario, 1Z, 4A

Sandfort, Veit, OK, 00

Savitz, Sean I., OD

Schellingerhout, Dawid, 2T

Schenke, Hendrik, 10

Schenone, Daniela, 1Z, 4A

Schlaefer, Alexander, 14, 1L

Schoon, Erik, 31

Schram, Miranda T., IN

Scott, Christopher G., OW

Selvam, Amrish, OV

Sensakovic, William F., OU

Seong, Jinsil, 3H, 3L

Shah, Jay, $1 \mathrm{M}$

Shah, Meet, 06

Shahedi, Maysam, 1R

Shan, Caifeng, 4B

Sharma, Umesh, 4D

Shen, Chen, 3V

Sheth, Sunil A., OD

Shi, Zhongchao, OL

Shim, Kyu Won, 2 z

Shiradkar, Rakesh, 0M, 3G

Shiraz Bhurwani, Mohammad Mahdi, 20

Shu, Hui-kuo, $2 Y$

Siddiqui, Adnan H., 2O, 2W

Silamut, Kamolrat, $1 Q$

Singh, Sadanand, 06

Snyder, Kenneth V., 20

Sommer, Kelsey, 4D

Song, Peilun, 4E

Song, Xueqin, 4E

Sóñora-Mengan, Alexander, 3T

Sopkovich, Jennifer, 15

Sriram, Sai Aditya, OK

Starosolski, Zbigniew A., 3M

Stehouwer, Coen D. A., IN

Stein, Sharon, OV

Stidham, Ryan W., 36

Struyvenberg, Maarten, 31, 37

Su, Grace L., 36

Su, Jason, 06

Su, Xiu, $3 Z$
Succio, Giulia, 4A

Sudkamp, Helge, 10

Suetens, Paul, 23

Sui, Jing, $3 X$

Sullivan, Ryan, 21

Summers, Ronald M., 07, 09, 0K, 00, $1 \mathrm{~J}$

Sun, Edward, ON

Sun, Xudong, 04

Sun, Ying, 3W

Sun, Yue, 4B

Suzuki, Hidenobu, 2D

Suzuki, Kojiro, 34

Suzuki, Yuki, 2A

Swiecicki, Albert, OR, 28, 3N

Syeda-Mahmood, Tanveer, $1 \mathrm{~K}$

T. T., Anusha Devi, 1R

Tagliafico, Alberto Stefano, 4A

Taj-Schaal, Nazhat, $1 \mathrm{M}$

Takimoto, Aitaro, 34

Tamez-Peña, Jose, 2R

Tan, Jiaxing, 1S, 3D

Tan, Jimin, OP

Tan, Tao, 4B

Tang, Xiangyang, 44

Tang, You-Bao, 09, $1 \mathrm{~J}$

Tang, Yu-Xing, 07, 09

Tavakoli, Meysam, $3 Y$

Teknos, Theodoros, $1 \mathrm{M}$

Thiele, Frank, 1B

Thiessen, J., 3F

Tian, Jiang, OL

Tian, Zhen, $2 \mathrm{X}$

Tirumani, Sree Harsha, $3 G$

Tiwari, Pallavi, 1W, 30

Tomiyama, Noriyuki, 2A

Tong, Yubing, 3U

Torigian, Drew A., $3 \mathrm{U}$

Torri, Lorenzo, 4A

Tsuboshita, Yukihiro, $1 \mathrm{X}$

Tsuchida, Takaaki, 2J

Tsue, Trevor, 06

Tushar, Fakrul I., 08, 1P

Tutino, Vincent, $2 \mathrm{~W}$

Twickler, Diane M., $1 R$

Uchida, Hiroo, 34

Udupa, Jayaram K., $3 \mathrm{U}$

Uemura, Tomoki, OE, OT, 35, 3C

Ueno, Yuta, 46

Vachani, Anil, 2G

Vachon, Celine, OW

Vahidy, Farhaan S., OD

van der Putten, Joost, 31, 37

van der Sommen, Fons, 31, 37

van der Velden, Bas H. M., 12

Van Dusen, Amy, 21

van Ginneken, Bram, $1 C$

van Greevenbroek, Marleen J., IN

Van Ongeval, Chantal, 23

Pul, Carola van, 4B

xviii

Proc. of SPIE Vol. 11314 1131401-18 
Vanbilsen, Chelsey, 23

Vandemeulebroucke, Jef, OS, 2F, 3T

Vandevenne, Jan, 23

Veasey, Benjamin, 29

Vellal, Adithya, 13

Verhage, Levi, 37

Verma, Ruchika, $1 \mathrm{~W}$

Veta, Mitko, $1 \mathrm{~N}$

Vidyaratne, Lasitha, $\mathrm{OH}$

Vieceli, Michael, 21

Viswanath, Satish E., OV

von der Burchard, Claus, 10

Wainwright, Jocelyn, 2G

Walsh, Ruth, 28

Wang, Caihua, $1 \mathrm{X}$

Wang, Chenglong, 0 I

Wang, David, 3B

Wang, Ge, 2C

Wang, Lili, 39

Wang, Stewart C., 36

Wang, Tonghe, 2Y, 3P, 40, 44, 45, 4F

Wang, Wenjin, $4 \mathrm{~B}$

Wang, Wensheng, $2 S$

Wang, Yaping, 4E

Wang, Yi, ON

Wang, Yifan, $1 \mathrm{~F}$

Wang, Yinyan, $2 M$

Wang, Yiyang, 48

Waqas, Muhammad, 2O, 2W

Ward, A. D., 3F

Wasnik, Ashish P., 36

Watari, Chinatsu, OT

Weddell, Rob, 48

Wei, Alice, 3B

Wei, Dong, $2 M$

Wei, Jun, 1F, 26, 3K, 3W

Wei, Leihao, $1 \mathrm{H}$

Weinberg, Jeffrey S., 2T

Weizer, Alon, 1T, 3K

Westermann, Stephan, $1 \mathrm{~L}$

Whitney, Heather M., OX, 11, 21

Wiemker, Rafael, 1B

Williams, Kyle A., 20

Wilson, Michael F., 4D

Winham, Stacey J., OW

Wismüller, Axel, 1U, 1Y

Wong, Ken C. L., 1K

Wu, Jie, 39

Wu, Shandong, $1 \mathrm{~A}$

WU, Yan, $0 Q$

$X i$, Yin, $1 R$

Xia, Yujiao, 41

Xiao, Jing, 09, $1 \mathrm{~J}$

Xie, Weiyang, $1 \mathrm{E}$

Xie, Zheng, 17

Xing, Lei, $O Q$

$X U$, Feiyu, OL

$X U$, Feng, $2 S$

$X U, X i n, 2 S$
Yamagata, Kazuki, 2A

Yamaguchi, Takefumi, 46

Yan, Pingkun, 2C

Yanagawa, Masahiro, 2A

Yang, Feng, 1Q

Yang, Guang, 39

Yang, Xiaofeng, 2X, 2Y, 3P, 40, 44, 45, 4F

Yankelevitz, David F., OA

Yerebakan, Halid, 2B

Yesha, Yelena, 2E

Yin, Yi, 4C

Yip, Rowena, OA

Yoon, Hong-Jun, 3R

Yoshida, Hiroyuki, OE, OT, 35, 3C

YU, David S., 44

YU, Hang, $1 Q$

Yu, Jennifer, 30

Yu, Lianbo, $1 \mathrm{M}$

Zamzmi, Ghada, 4G

Zanella-Calzada, Laura A., 24

Zeng, Johnathan, 30

Zhang, Bofei, OP

Zhang, Long Jiang, 3P, 40, 45, 4F

Zhang, Shu, 1S, 3B, 3D, 3E

Zhang, Zhenfeng, 3Q

Zhao, Jun, 1E

Zhao, Yiyuan, 2B

Zhao, Yu, 30

Zheng, Bin, 39

Zheng, Yefeng, $2 M$

Zhong, Cheng, OL

Zhou, Chuan, 1F, 1T, 26, 3K

Zhou, Yuanpin, 26

Zhu, Yingying, OK

Zinger, Svitlana, 31

Zong, Rui, $2 S$

Zuo, Ruyuan, 3G

Zurada, Jacek, 29 


\title{
Conference Committee
}

\author{
Symposium Chairs
}

Georgia D. Tourassi, Oak Ridge National Laboratory (United States)

Metin N. Gurcan, Wake Forest Baptist Medical Center (United States)

Conference Chairs

Horst K. Hahn, Fraunhofer MEVIS (Germany) and Jacobs Universität Bremen (Germany)

Maciej A. Mazurowski, Duke University (United States)

Conference Program Committee

Sameer K. Antani, U.S. National Library of Medicine (United States)

Samuel G. Armato III, The University of Chicago (United States)

Susan M. Astley, The University of Manchester (United Kingdom)

Ulas Bagci, University of Central Florida (United States)

Matthew S. Brown, University of California, Los Angeles (United States)

Heang-Ping Chan, University of Michigan (United States)

Weijie Chen, U.S. Food and Drug Administration (United States)

Marleen de Bruijne, Erasmus MC (Netherlands)

Thomas M. Deserno, Technische Universität Braunschweig (Germany)

Karen Drukker, The University of Chicago (United States)

Jan Ehrhardt, Universität zu Lübeck (Germany)

Catalin Fetita, Télécom SudParis (France)

Hiroshi Fujita, Gifu University School of Medicine (Japan)

Maryellen L. Giger, The University of Chicago (United States)

Hayit Greenspan, Tel Aviv University (Israel)

Lubomir M. Hadjiiski, University of Michigan (United States)

Takeshi Hara, Gifu University School of Medicine (Japan)

Helen Hong, Seoul Women's University (Republic of Korea)

Khan M. Iftekharuddin, Old Dominion University (United States)

Nico Karssemeijer, Radboud Universiteit Nijmegen Medisch Centrum (Netherlands)

JongHyo Kim, Seoul National University Hospital (Korea, Republic of)

Zhengrong Jerome Liang, Stony Brook University (United States)

Marius George Linguraru, Children's National Medical Center

(United States)

Fabrice Meriaudeau, Université de Bourgogne (France)

Kensaku Mori, Nagoya University (Japan)

Janne J. Näppi, Massachusetts General Hospital (United States) and

Harvard Medical School (United States)

Noboru Niki, University of Tokushima (Japan) 
Carol L. Novak, Siemens Healthineers (United States)

Nicholas A. Petrick, U.S. Food and Drug Administration (United States)

Letícia Rittner, Universidade Estadual de Campinas (Brazil)

Clarisa I. Sánchez, Radboud Universiteit Nijmegen Medisch Centrum (Netherlands)

Ronald M. Summers, National Institutes of Health (United States)

Kenji Suzuki, Illinois Institute of Technology (United States)

Pallavi Tiwari, Case Western Reserve University (United States)

Georgia D. Tourassi, Oak Ridge National Laboratory (United States)

Rafael Wiemker, Philips Research (Germany)

Axel Wismüller, University of Rochester Medical Center (United States)

Shandong Wu, University of Pittsburgh (United States)

Xiaofeng Yang, Emory University (United States)

Hiroyuki Yoshida, Massachusetts General Hospital (United States) and Harvard Medical School (United States)

Chuan Zhou, University of Michigan Health System (United States)

Session Chairs

1 Mammography

Georgia Tourassi, Oak Ridge National Laboratory (United States)

Horst K. Hahn, Fraunhofer-Institut für Digitale Medizin MEVIS

(Germany)

2 Chest I

Matthew S. Brown, University of California, Los Angeles (United States)

Samuel G. Armato III, The University of Chicago (United States)

3 Neuro I

Letícia Rittner, Universidade Estadual de Campinas (Brazil)

Khan M. Iftekharuddin, Old Dominion University (United States)

4 Abdomen

Kenji Suzuki, Illinois Institute of Technology (United States)

Hiroyuki Yoshida, Massachusetts General Hospital (United States)

5 Musculoskeletal

Zhengrong Jerome Liang, Stony Brook University (United States)

Axel Wismüller, University of Rochester Medical Center (United States)

6 Radiomics

Kensaku Mori, Nagoya University (Japan)

Heang-Ping Chan, Michigan Medicine (United States) 
7 Breast MRI, Skin

Maryellen L. Giger, The University of Chicago (United States)

Thomas Martin Deserno, Technische Universität Braunschweig (Germany)

8 Breast

Shandong Wu, University of Pittsburgh (United States)

Karen Drukker, The University of Chicago Medicine (United States)

9 Chest II, Lymph Nodes

Ronald M. Summers, National Institutes of Health Clinical Center (United States)

Fujii Hiroshi, Gifu University (Japan)

10 Keynote and Methodology

Horst K. Hahn, Fraunhofer-Institut für Digitale Medizin MEVIS (Germany)

Maciej A. Mazurowski, Duke University (United States)

11 Head and Neck, Eye

Maciej A. Mazurowski, Duke University (United States)

Carol L. Novak, Siemens Healthineers (United States)

12 Novel Applications

Lubomir M. Hadjiiski, Michigan Medicine (United States)

Sameer K. Antani, U.S. National Library of Medicine (United States)

13 Neuro II

Pallavi Tiwari, Case Western Reserve University (United States)

Maciej A. Mazurowski, Duke University (United States) 
Proc. of SPIE Vol. 11314 1131401-23

Downloaded From: https://www.spiedigitallibrary.org/conference-proceedings-of-spie on 25 Apr 2023 Terms of Use: https://www.spiedigitallibrary.org/terms-of-use 


\section{Medical Imaging Award Recipients}

\section{Robert F. Wagner Best Student Paper Award}

Robert F. Wagner was an active scientist in the SPIE Medical Imaging meeting, starting with the first meeting in 1972 and continuing throughout his career. He ensured that the BRH, and subsequently the CDRH, was a sponsor for the early and subsequent Medical Imaging meetings, helping to launch and ensure the historical success of the meeting. The Robert $F$. Wagner All-Conference Best Student Paper Award (established 2014) is acknowledgment of his many important contributions to the Medical Imaging meeting and his many important advances to the field of medical imaging.

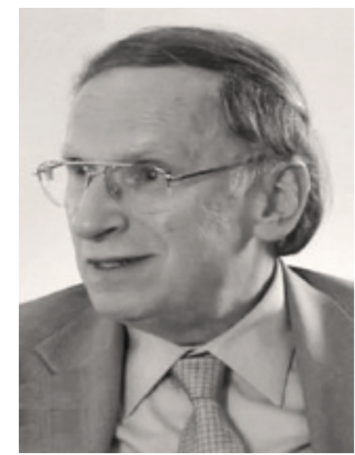

This award is co-sponsored by:

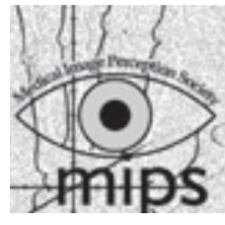

The Medical Image Perception Society

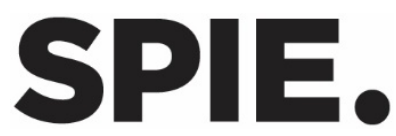

2020 Recipients:

First Place: Multi-body registration for fracture reduction in orthopaedic trauma surgery (11315-14)

R. Han, A. Uneri, P. Wu, R. Vijayan, P. Vagdargi, M. Ketcha, N. Sheth, Johns Hopkins University (United States), S. Vogt, G. Kleinszig, Siemens Healthineers (Germany) G. M. Osgood, John Hopkins Hospital (United States), J. H. Siewerdsen, John Hopkins University (United States)

Second Place: Phase contrast CT enabled three-material decomposition in spectral CT imaging (11312-47)

Xu Ji, Ran Zhang, Ke Li, Guang-Hong Chen, University of Wisconsin School of Medicine and Public Health (United States) 
Proc. of SPIE Vol. 11314 1131401-25

Downloaded From: https://www.spiedigitallibrary.org/conference-proceedings-of-spie on 25 Apr 2023 Terms of Use: https://www.spiedigitallibrary.org/terms-of-use 